\title{
Das Krossenrezidiv aus der Sicht des Operateurs
}

\section{Saphenofemoral recurrences from a surgical point of view}

Autoren

Achim Mumme ${ }^{1,2}$, Dominic Mühlberger ${ }^{1,2}$, Thomas Hummel ${ }^{1,2}$

Institute

1 Klinik für Gefäßchirurgie, St.-Josef-Hospital, Katholisches Klinikum Bochum der Ruhr-Universität Bochum

2 Venenzentrum der Dermatologischen und Gefäßchirurgischen Kliniken des Katholischen Klinikums Bochum der Ruhr-Universität Bochum

\section{Schlüsselwörter}

Varikose, Krossektomie, Langzeitergebnisse,

Barriere-Techniken

\section{Key words}

varicose vein disease, flush ligation, long term results, barrier techniques

\section{Bibliografie}

DOI https://doi.org/10.1055/a-1163-1057

Online-Publikation: 25.5.2020

Phlebologie 2020; 49: 139-143

(c) Georg Thieme Verlag KG, Stuttgart · New York ISSN 0939-978X

\author{
Korrespondenzadresse \\ Prof. Dr. Achim Mumme \\ Klinik für Gefäßchirurgie, St.-Josef-Hospital, Katholisches \\ Klinikum Bochum der Ruhr-Universität Bochum, \\ Gudrunstr. 56, 44791 Bochum \\ Achim.mumme@ruhr-uni-bochum.de
}

\section{ZUSAMMENFASSUNG}

Krossenrezidive nach Varizenoperation haben eine hohe sozioökonomische Relevanz. Zur Rezidivprophylaxe steht die
Vermeidung technischer Fehler beim Ersteingriff im Vordergrund. Es darf kein Saphenastumpf verbleiben, der später zum proximalen Insuffizienzpunkt einer Rezidivvarikose werden kann. Darüber hinaus sollte die Durchtrittsstelle für saphenofemorale Rezidive, das Foramen ovale, beim Ersteingriff verschlossen werden, um eine Faszienbarriere zwischen der tiefen Vene und dem oberflächlichen Kompartiment zu schaffen. Bei Rezidivoperationen mit Vernarbungen im Bereich des Foramen ovale kann die Herstellung einer Faszienbarriere durch eine Augmentierung mit einem Flicken erleichtert werden.

Mit einer ordnungsgemäßen Krossektomie in Kombination mit einer Faszienbarriere können Krossenrezidive weitgehend vermieden werden.

\section{ABSTRACT}

Saphenofemoral recurrences after varicose vein surgery have a high socio-economic relevance. Prevention of recurrence is focused on avoiding technical errors during the first intervention. No saphenous stump may remain, which can later become the source of reflux for recurrent varicosis. In addition, the passage point for saphenofemoral recurrences, the foramen ovale, should be closed during the first procedure to create a fascia barrier between the deep vein and the superficial compartment. In the case of redo-surgery with scarring in the area of the oval foramen, the creation of a fascia barrier can be facilitated by augmentation with a patch.

With a proper crossectomy in combination with a fascia barrier, saphenofemoral recurrences can be largely avoided.

\section{Einleitung}

Ca. 80 \% der Rezidive nach Krossektomie und Stripping der Vena saphena magna werden durch Insuffizienzen aus dem Krossenbereich gespeist. Dementsprechend kommt der Krossenregion eine Schlüsselfunktion zu, wenn es um die Vermeidung von Rezidiven geht. Dieser Zusammenhang war bereits in den 60er-Jahren phlebochirurgischen Pionieren wie Nabatoff oder Dodd bekannt [1, 20]. In größeren Fallserien konnten die Autoren nachweisen, dass die inkomplette Entfernung der VSM mit Belassen eines Krossestumpfes zur Ausbildung von Rezidiven führt. Dieser Erkenntnis folgend wurde das Prinzip der Krossektomie entwickelt: die
Resektion der krankhaft veränderten VSM im Krossenbereich mit bündiger Ligatur des Gefäßes an der Vena femoralis communis. Das Motto „No stump, no recurrence“ ist seither zu einem phlebochirurgischen Leitsatz geworden, der in venenchirurgischen Lehrbüchern [11] und in den Leitlinien der Deutschen Gesellschaft für Phlebologie Berücksichtigung findet [21].

Krossenrezidive können aber auch nach technisch einwandfreier Operation auftreten. Ausgehend von der Ligaturstelle an der tiefen Vene beeinträchtigen sogenannte Neovaskulate das Operationsergebnis. In einem noch weitgehend unerforschten Prozess kommt es infolge der Neubildungen zur Wiederherstellung der unerwünschten Passage vom tiefen in das oberflächliche Venen- 

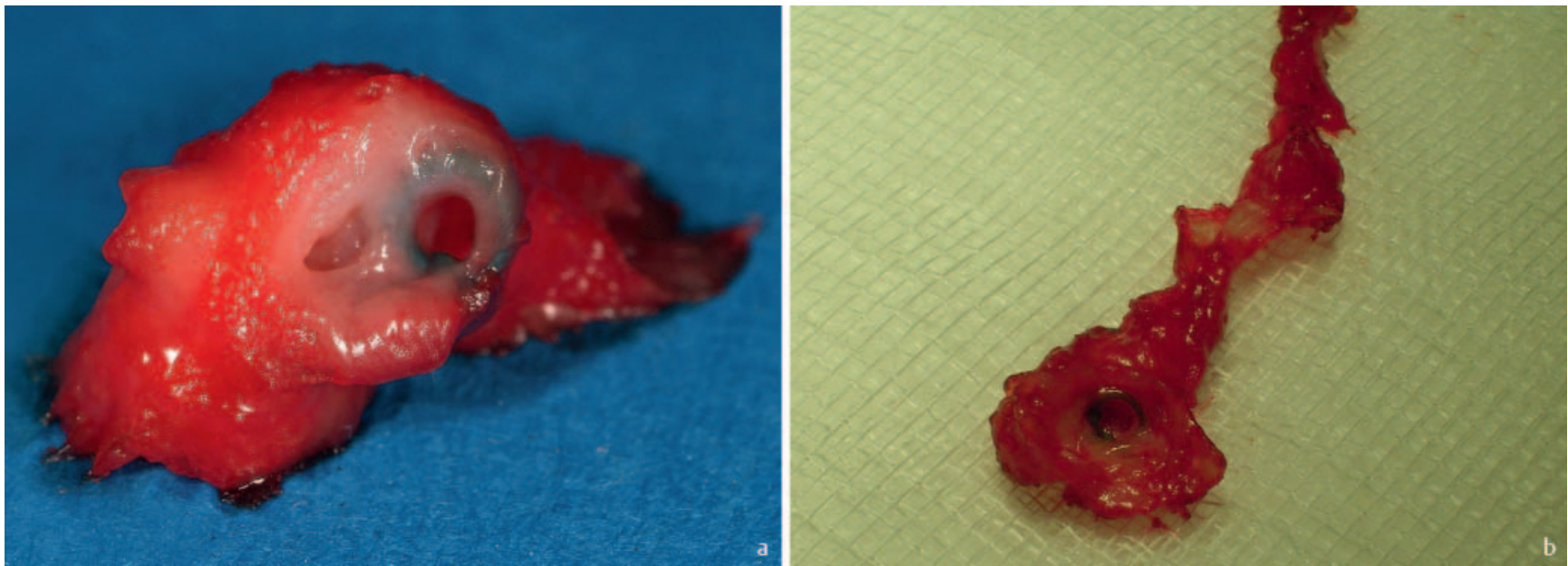

- Abb.1 Resektate von saphenofemoralen Rezidiven mit Rekanalisation des ligierten Saphenastumpfes und anschließender Neovaskularisation. a Präparat mit rekanalisierter Ligatur (Mersilene R), Resektionsrand an der V. femoralis communis. b Dünnwandiges und geschlängeltes Neovaskulat, das sich an einem rekanalisierten Saphenastumpf entwickelt hat.

system [3]. Ab einem Durchmesser von ca. 5 mm werden die Refluxwege in der Krosse klinisch relevant und können zum proximalen Insuffizienzpunkt einer Rezidivvarikose werden.

Die Neovaskulate entspringen direkt neben der ursprünglichen Ligaturstelle als Mono-Channel oder Multi-Channel-Rezidiv [2]. Sie können aber auch mit einer Rekanalisation des ligierten Saphenastumpfes einhergehen. In diesem Fall schließt sich das Neovaskulat direkt an den Resektionsrand der Vene an ( $\triangleright$ Abb. 1a, b).

Die klinische Bedeutung der Neovaskularisation ist bis dato nicht eindeutig geklärt. In ultraschallbasierten Studien wurden Neovaskularisationsraten von bis zu $60 \%$ beschrieben. Diese Studien waren jedoch methodisch fragwürdig, nachdem ein duplexsonografischer Nachweis von Neovaskulaten nur eingeschränkt möglich ist [6]. Dennoch wurde die Neovaskularisation vielfach als Hauptursache des Krossenrezidivs angesehen [3, 18, 23] und damit die Sinnhaftigkeit einer operativen Krossektomie infrage gestellt.

Mit der Deutschen Leistenrezidiv-Studie [7, 16, 18] erfolgte erstmalig eine histopathologische und immunhistochemische Analyse der Rezidivursachen in einem großen Kollektiv. An 7 Zentren für Varizenchirurgie wurde bei 427 Patienten anlässlich der Reoperation das Rezidivgefäß exstirpiert und zur feingeweblichen Untersuchung eingeschickt. Nur 16,2\% ( $n=69)$ der Rezidive waren tatsächlich auf ein Neovaskulat zurückzuführen. Dagegen zeigte sich bei 65,3\% ( $n=279)$ der Präparate ein zu lang belassener Saphenastumpf als Rezivursache. Nicht die Neovaskularisation, sondern die technisch fehlerhafte Krossektomie ist also die Hauptursache des Leistenrezidivs. Im Umkehrschluss bestätigte die Studie den phlebochirurgischen Grundsatz, dass die ordnungsgemäße Krossektomie ein Leistenrezidiv weitestgehend verhindert.

Zur Überprüfung dieser These wurde von der Arbeitsgemeinschaft Varizenoperationen der Deutschen Gesellschaft für Phlebologie die LaVaCro-Studie initiiert [22]. An 12 phlebochirurgischen Zentren wurden 841 Patienten mit 1070 standardisiert durchgeführten Krossektomie- und Stripping-Operationen in die Studie aufgenommen. Nur bei 2,24\% der prospektiv untersuchten Extre- mitäten zeigte sich im Verlauf nach 1 Jahr ein duplexsonografisch verifizierbarer Krossenreflux, der nur in 0,29\% der Fälle klinisch relevant war. Damit lieferte auch die LaVaCro-Studie eine Bestätigung der alten phlebochirurgischen Weisheit: Die ordnungsgemäße Krossektomie verhindert weitestgehend das Rezidiv.

\section{Rezidivprophylaxe durch eine ordnungsgemäße Krossektomie}

Die ultraschallassistierte Operationsplanung mit Anzeichnung der Refluxwege durch den Operateur ist ein wichtiges Qualitätskriterium für eine ordnungsgemäße Krossektomie.

Anatomische Varianten werden bereits präoperativ aufgedeckt und können damit in die Operationsplanung einbezogen werden. Im Bedarfsfall muss auch intraoperativ auf den Ultraschall zurückgegriffen werden können.

Selbstverständlich ist eine atraumatische Operationstechnik mit schonender Präparation erforderlich. Das Subkutangewebe wird mit einem glatten Schnitt bis zur Camper-Faszie durchtrennt. Diese wird anschließend längs gespalten. Bei der dann folgenden Präparation im lymphatischen Gewebe wird nur noch in Längsrichtung präpariert. Die Durchtrennung von Lymphbahnen muss vermieden werden. Es folgt die Dissektion der VSM und ihrer Seitenäste. Dabei werden auch das Foramen ovale und die Vorderwand der tiefen Vene dargestellt $(\checkmark \mathbf{A b b}$. 2a). Nach Absetzen der Seitenäste wird die VSM in Höhe der Mündungsklappe abgeklemmt ( $\triangleright$ Abb. $\mathbf{2 b}$ ). Die VSM wird anschließend abgesetzt und mit nicht resorbierbarem Nahtmaterial im Niveau der tiefen Vene ligiert. Zur Prophylaxe einer Neovaskularisation sollte das an der Stumpfrosette freiliegende Endothel der VSM ausgeschaltet werden. Hierzu verschorfen wir vorsichtig das Endothel mit dem Elektrokauter. Anschließend führen wir noch eine Invertierungsplastik nach Frings [5] durch. Mit einer überwendlich gestochenen Naht wird dabei die Stumpfrosette verschlossen und damit das Endothel der Vene bedeckt. ( $\vee$ Abb. 2c). Ein weiterer wichtiger Operationsschritt ist der Verschluss des Foramen ovale mit einer Naht 

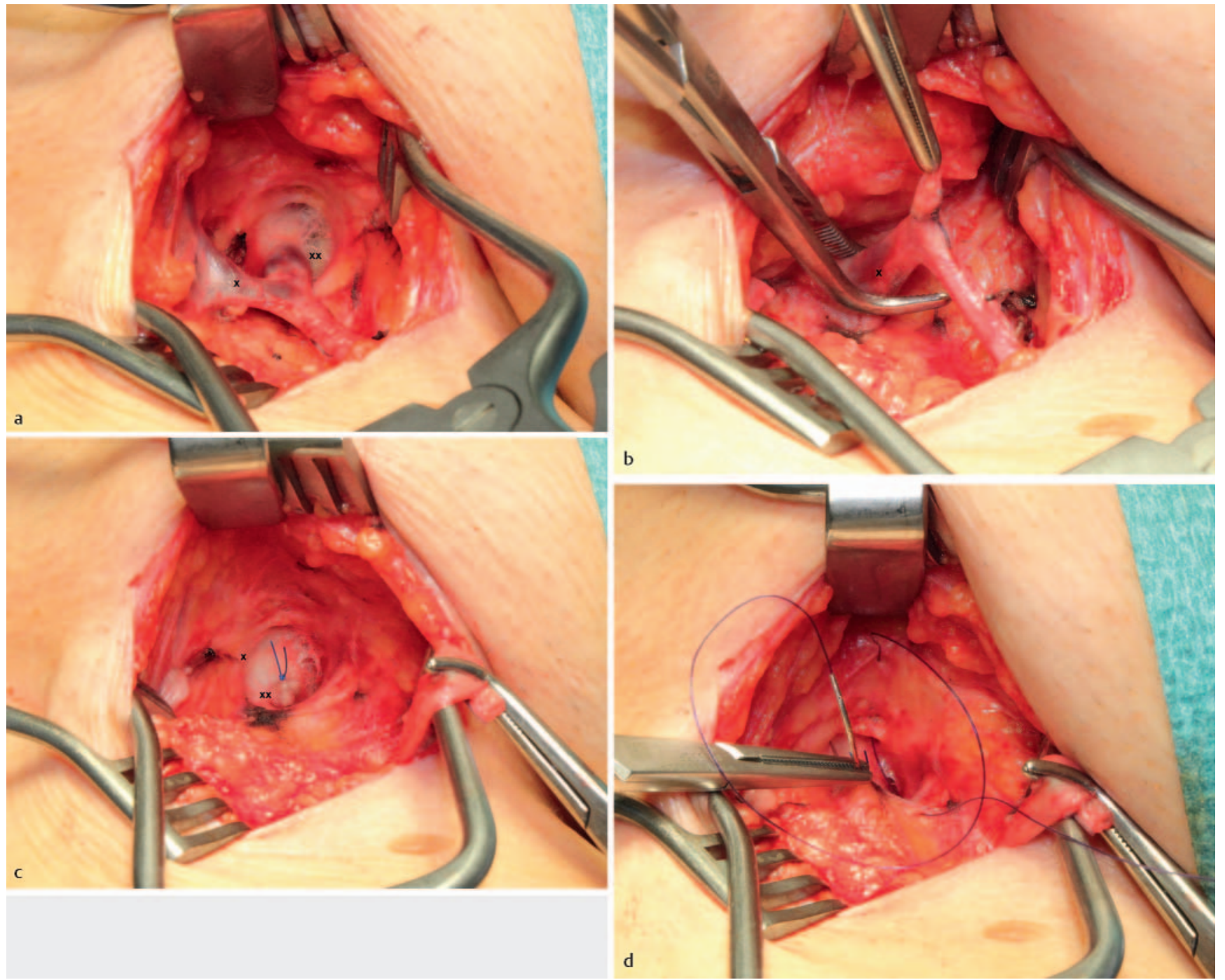

- Abb.2 a Einmündungsbereich der Saphenakrosse mit der Einmündung der V. epigastrica superficialis (X). Blick auf das Foramen ovale der Fascia lata (XX). Direkt unterhalb der Faszie liegt die V. femoralis communis. b Eine Overholt-Klemme wird im Niveau der Mündungsklappe gesetzt. c Blick in das Foramen ovale $(\mathrm{XX})$ nach erfolgter Krossektomie und Invertierungsplastik des Endothels nach Frings. $\mathbf{d}$ Verschluss des Foramen ovale mit resorbierbarem Nahtmaterial. Der Saphenastumpf bleibt unterhalb der Faszia lata. Die Schaffung einer Faszienbarriere dient der Prophylaxe von Krossenrezidiven. Quelle: Rabe E, Stücker M (Hrsg). Phlebologischer Bildatlas. WPV. Verlag, Köln 2015. [rerif]

( $\triangleright$ Abb.2 d). Diese Maßnahme dient ebenfalls der Rezidivprophylaxe. Schließlich nehmen sämtliche saphenofemoralen Rezidive ihren Weg durch das Foramen hindurch. Der Faszienverschluss soll eine Barriere bilden und das tiefe Kompartiment vom oberflächlichen dauerhaft trennen. De Maeseneer et al. [15] konnten im Vergleich zu einer historischen Kontrollgruppe einen signifikanten Vorteil der Barriere-Technik zeigen mit einer Reduktion der inguinalen Neorefluxraten im 1-Jahres-Follow-up von 14,8\% auf $6,7 \%$.

\section{Operative Therapie des inguinalen Krossenrezidivs}

Der Zugang zum saphenofemoralen Rezidiv kann über verschiedene Wege gewonnen werden [8-10, 12, 13]. Die am häufigsten gebräuchlichen Methoden sind die präfemorale Stumpfligatur nach Hach [10] und der modifizierte Zugang nach Junod [12]. Bei beiden Methoden wird das Narbengewebe der Voroperation umgangen, und zwar von proximal bei der von Hach beschriebenen Methode und von lateral bei dem Zugang nach Junod. Nach unserer Erfahrung bietet der laterale Zugang Vorteile im Hinblick auf die Schonung des lymphatischen Gewebes, das umgangen wird.

Ausgehend von einer Inzision in der Leistenfalte erfolgt nach der Durchtrennung des Fettgewebes die Darstellung der Camper-Faszie, welche das lymphatische Gewebe bedeckt. Nun wird nur noch in Längsrichtung präpariert. Die Längsdurchtrennung des lymphatischen Gewebes erfolgt dann 1-2 Querfinger lateral des Leistenpulses. Dort läuft das lymphatische Gewebe aus. Wesentliche Schädigungen des Lymphapparates sind hier nicht zu erwarten. Nach Darstellung der Faszie ändert sich die Präparationsrichtung nach medial. Unter atraumatischer Abhebung des 
Lymphgewebes wird über die pulsierend tastbare Arterie hinweg präpariert bis an den Rand des meist vernarbten Foramen ovale, wo sich das saphenofemorale Rezidiv befindet. Die Faszie wird längs geöffnet und die Vorderwand der V. femoralis communis dargestellt. Unter Beachtung der hier meist quer verlaufenden A. pudenda externa wird das Rezidiv von subfaszial aus in das epifasziale Gebiet hinein zirkulär freipräpariert, bis das Gefäß klemmbar ist und durchtrennt werden kann. Der proximale Gefäßstumpf wird analog zum Ersteingriff mit nicht resorbierbarer Naht versorgt. Der distale Stumpf wird ebenso übernäht. Gegebenenfalls kann nach distal hin auch noch eine Schaumsklerosierung von chirurgisch schlecht erreichbaren Konvoluten angeschlossen werden.

\section{Rezidivprophylaxe mit der Barriere-Operation}

Dem Verschluss der Faszienlücke im Foramen ovale kommt bei der Rezidivoperation eine besondere Bedeutung zu. Wegen der üblicherweise nach Voroperation bestehenden narbigen Veränderungen ist allerdings der Verschluss der Faszienlücke durch eine einfache Naht oft nicht möglich. In dieser Situation kann die Durchtrittspforte für saphenofemorale Rezidive mit der sogenannten Barriere-Operation verschlossen werden. Ein $3 \times 2 \mathrm{~cm}$ durchmessender Flicken aus Polytetrafluorethylen wird zentral auf das ligierte Saphenastumpfrezidiv aufgenäht. Hierzu dient eine $5 \times 0$-Naht aus Polypropylen. Die 4 Ecken des Flickens werden ebenfalls mit Polypropylennähten an der Fascia lata angeheftet, sodass eine strikte Trennung der tiefen Vene vom oberflächlichen Kompartiment resultiert ( $\vee$ Abb. $\mathbf{3}$ ).

\section{Diskussion}

Dreh- und Angelpunkt für die Nachhaltigkeit einer Varizenoperation ist die Krossenregion. Hier gilt es, eine strikte Trennung zwischen dem tiefen und dem oberflächlichen Kompartiment dauerhaft herbeizuführen. Die ordnungsgemäße Krossektomie ist dabei ein entscheidender Faktor. Es muss unbedingt vermieden werden, einen Stumpf der krankhaft veränderten V. saphena magna in situ zu belassen. Jeder Stumpf - auch ein noch so kurzer - ragt durch das Foramen ovale hindurch in das oberflächliche Kompartiment hinein und kann damit zum proximalen Insuffizienzpunkt eines Varizenrezidivs werden ( $\triangleright$ Abb. 4 ).

Die potenzielle Durchtrittspforte für das spätere saphenofemorale Rezidiv - das Foramen ovale - sollte im Rahmen der Krossektomie verschlossen werden. Auf diese Weise kann eine dauerhafte Faszienbarriere zwischen den Kompartimenten hergestellt werden. In der bereits erwähnten Multicenterstudie von Papapostolou et al. [22] konnte gezeigt werden, dass eine ordnungsgemäße Krossektomie in Verbindung mit dem Faszienverschluss zu guten Langzeitergebnissen führt.

Die strikte Trennung der Kompartimente ist auch bei der Rezidivoperation ein wesentliches Ziel, das allerdings meistens nur durch eine Art Bruchlückenverschluss, augmentiert mit einem Flicken aus Polytetrafluorethylen, erreicht werden kann. Die Wirksamkeit der sogenannten Barriere-Operation wurde bereits durch eigene [4] und internationale Studien [14, 24] belegt.

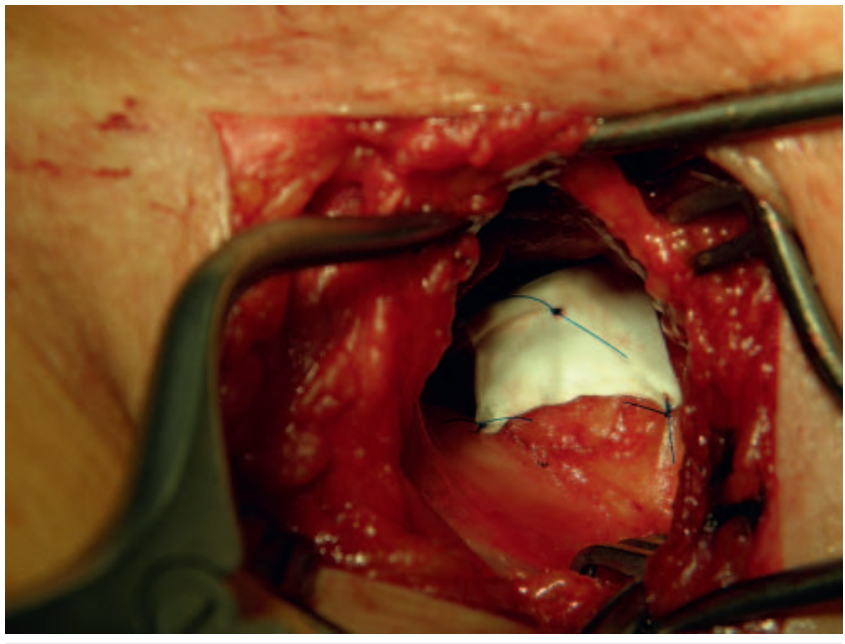

- Abb. 3 Verschluss des Foramen ovale der Fascia lata mit einem $3 \times 2 \mathrm{~cm}$ durchmessenden Flicken aus Polytetrafluorethylen. Dadurch soll die Faszienbarriere zwischen dem tiefen und dem oberflächlichen Kompartiment komplettiert werden.

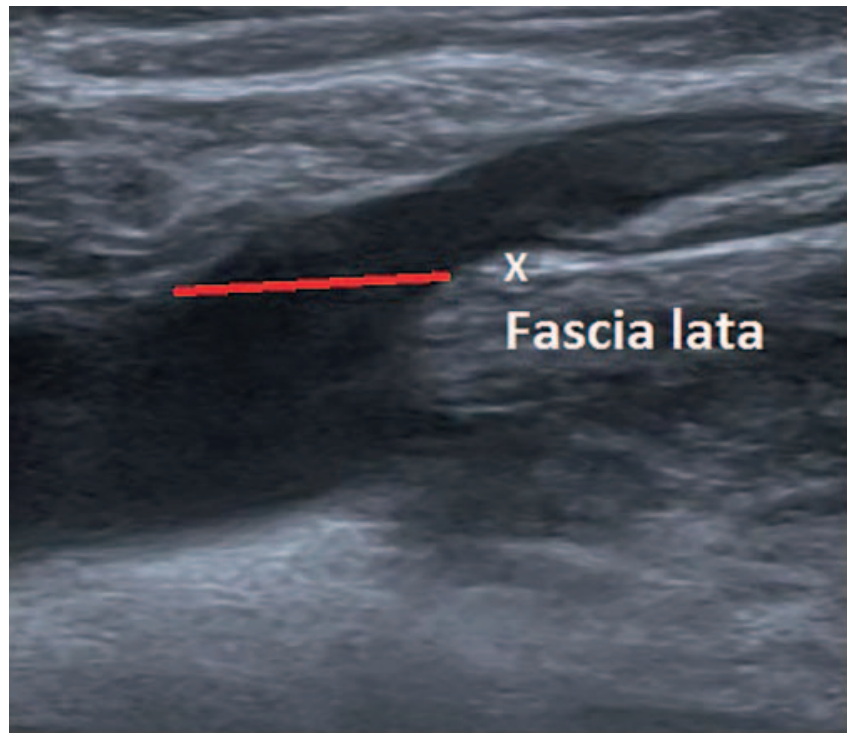

- Abb.4 Sonografische Darstellung der V. saphena magna-Krosse. Die Fascia lata liegt der tiefen Vene unmittelbar auf. Auch ein kurzer Stumpf würde in das oberflächliche Kompartiment (rote Linie) hineinragen.

Die aus der operativen Rezidivforschung gewonnenen Erkenntnisse gelten grundsätzlich auch für die endovenösen Verfahren. Die im Vergleich zur operativen Therapie höhere Rate saphenofemoraler Rezidive [17, 19, 25] hängt sehr wahrscheinlich damit zusammen, dass die dauerhafte Trennung der Kompartimente allein auf endovenösem Wege problematisch ist. Analog zu den Leitlinienempfehlungen für die operative Behandlung müsste ein endovenöser Krossenverschluss unterhalb vom Niveau der Fascia lata bleiben. Inwieweit dies technisch möglich ist, muss durch weitergehende Studien geklärt werden. 


\section{Interessenkonflikt}

Die Autorinnen/Autoren geben an, dass kein Interessenkonflikt besteht.

\section{Literatur}

[1] Dodd H. Persistent or recurrent varicose veins. Br J Clin Pract 1963; 17 : 501-505

[2] Fischer R, Linde N, Duff C et al. Late recurrent saphenofemoral junction reflux after ligation and stripping of the greater saphenous vein. J Vasc Surg 2001; 34: 236-240

[3] Fischer R, Chandler NJG, De Maesener MG et al. Collective review: the unresolved problem of recurrent saphenofemoral reflux. J Am Coll Surg 2002; 195: 71-85

[4] Freis H, Geier B, Mumme A et al. Barrier patch implantation during redo surgery for varicose vein recurrences in the groin: 1-year results. Ann Vasc Surg 2016; 35: 98-103

[5] Frings N, Brümmer L, Prinz N et al. Possibilities to prevent varicose veins after surgery. Phlebologie 2019; 48: 32-38

[6] Geier B, Mumme A, Hummel T et al. Validity of duplex-ultrasound in identifying the cause of groin recurrence after varicose vein surgery. J Vasc Surg 2005; 49: 968-972

[7] Geier B, Hummel T, Burger P et al. Residual stumps as a cause for inguinal varicose vein recurrences: A multicentre study. Eur J Vasc Endovasc Surg 2008; 36 (2): 207-210

[8] Gerontopoulou SA, Kath W, Rass K. Short-Term Efficacy of Inguinal Reoperation for Recurrent Saphenofemoral Incompetence using the Stump Suture Technique. Ann Vasc Surg 2018; 53: 197-204

[9] Gerontopoulou SA, Rass K. Therapie des inguinalen Crossenrezidivs: Ist die offene Re-Crossektomie noch zeitgemäß? Phlebologie 2019; 48 (1): 39-46

[10] Hach W. Zugangswege für die präfemorale Ligatur und Dissektion des kurzen Saphenastumpfes bei der Operation der Rezidivvarikose. Gefäßchirurgie 1996; 1: 56-57

[11] Hach W, Mumme A, Hach-Wunderle V (Hrsg) VenenChirurgie. 3. Auflage Stuttgart: Schattauer-Verlag; 2013

[12] Junod JM. Varices et leurs complications: traitement chirurgical des cas difficiles. Helv Chir Acta 1971; 1: 167-170

[13] Luke JC. The surgical treatment of recurrent varicose veins. Can J Surg 1958; 2 (1): 51-55
[14] De Maeseneer MG, Vandenbroeck CP, Van Schil PE. Silicone patch saphenoplasty to prevent repeat recurrence after surgery to treat recurrent saphenofemoral incompetence: Long-term follow-up study. J Vasc Surg 2004; 40 (1): 98-105

[15] De Maeseneer MG, Philipsen TE, Vandenbroeck CP et al. Closure of the Cribriform Fascia: An Efficient Anatomical Barrier Against Postoperative Neovascularisation at the Saphenofemoral Junction? A Prospective Study. Eur J Vasc Endovasc Surg 2007; 34: 361-366

[16] Mumme A, Olbrich S, Babera L et al. Saphenofemorales Leistenrezidiv der Vena saphena magna: technischer Fehler oder Neorevaskularisation? Phlebologie 2002; 31: 38-41

[17] Mumme A, Burger P, Hummel T et al. Der lang belassene Saphenastumpf - Implikationen für die endovenöse Therapie der Varikose. Phlebologie 2007; 36: 256-259

[18] Mumme A, Hummel T, Burger P et al. High ligation of the saphenofemoral junction is necessary. Results of the German Groin Recurrence Study. Phlebologie 2009; 38: 99-102

[19] Mumme A, Mühlberger D, Sidhwa Y et al. Alarmingly high rate of saphenofemoral recurrence following endovenous laser treatment. Phlebologie 2019; 48: 18-22

[20] Nabatoff RA. Reasons for major recurrence following operations for varicose veins. Surg Gyn \& Obst 1969; 128: 275-278

[21] Pannier F, Noppeney T, Alm J et al. S2k - Leitlinie Diagnostik und Therapie der Varikose Stand 03/19 AWMF-Register Nr. 037/018. AWMF-online 2019

[22] Papapostolou G, Altenkämper H, Bernheim C. Die LaVaCro-Studie: Langzeitergebnisse der Varizenoperation mit Crossektomie und Stripping der V. saphena magna. Interimsergebnisse nach 1 Jahr. Phlebologie 2013; 42 (5): 253-260

[23] Van Rij AM, Jones GT, Hill GB. Neovascularisation and recurrent varicose veins: More histologic and ultrasound evidence. J Vasc Surg 2004; 40: 296-302

[24] Van Rij AM, Jones GT, Hill BG et al. Mechanical Inhibition of Angiogenesis at the Saphenofemoral Junction in the Surgical Treatment of Varicose Veins Early Results of a Blinded Randomized Controlled Trial. Circulation 2008; 118: 66-74

[25] Rass K. Crossektomie und Stripping vs. endothermische Ablation der V. saphena magna: Was können wir aus aktuellen Langzeitanalysen lernen? Phlebologie 2018; 47: 265-271 